\title{
Factor structure and external correlates of posttraumatic stress disorder symptoms among African American firefighters
}

This article was published in the following Dove Press journal:

Psychology Research and Behavior Management

II August 2016

Number of times this article has been viewed

\author{
Consuelo Arbona \\ Weihua Fan \\ Nausheen Noor \\ Department of Psychological, Health, \\ and Learning Sciences, University of \\ Houston, Houston, TX, USA
}

\begin{abstract}
This study compared the relative goodness of fit of three well-established factorial models of posttraumatic stress disorder (PTSD) symptoms among 477 African American male firefighters in a large city in the US. The compared models were the two four-factor emotional numbing and dysphoria models and a five-factor dysphoric arousal model. The study also examined the convergent and discriminant validity of PTSD symptom clusters in relation to depression and alcohol dependence symptoms. Both the emotional numbing and dysphoric arousal PTSD models provided a superior fit to the data compared to the dysphoria model. Findings also indicated a good fit for factor models that included PTSD, depression, and alcohol dependence latent factors, which provides support for the specificity of PTSD symptom clusters. Depression symptoms were more strongly correlated with PTSD symptom clusters than alcohol dependence. In the dysphoric arousal model, depression and alcohol dependence were equally related to the emotional numbing and dysphoric arousal clusters; however, both depression and alcohol dependence were more highly correlated with dysphoric arousal than with anxious arousal. Even though the emotional numbing and dysphoric arousal models demonstrated a superior fit to the data, the four-factor dysphoria model may provide a more parsimonious representation of PTSD's latent structure than the five-factor dysphoric arousal model. In conclusion, this study extends support for the well-established PTSD symptom factor models among African Americans, a population with whom these models had not been examined earlier.
\end{abstract}

Keywords: posttraumatic stress disorder symptoms, PTSD, African American, factor structure, depression, alcohol problems

\section{Introduction}

Posttraumatic stress disorder (PTSD) is a multisymptom chronic disorder that was first identified in combat veterans and rape victims. Currently, PTSD is also recognized among those who witness traumatic events or care for those who have experienced trauma such as paramedics and firefighters. ${ }^{1,2}$ PTSD symptoms include intrusive re-experiencing of the traumatic event, avoidance of reminders of the situation, emotional numbing, and hyperarousal. ${ }^{3}$ Findings from national surveys are mixed regarding the relative incidence of PTSD among African American and non-Hispanic White adults. Although some studies report no differences in PTSD across ethnic groups, ${ }^{4}$ other studies indicate higher levels of PTSD among African Americans. In the latter studies, estimates of lifetime prevalence of PTSD have ranged from $8.7 \%$ to $9.1 \%$ among African Americans compared to $6.8 \%$ to $7.4 \%$ among non-Hispanic Whites. ${ }^{5,6}$ Studies with populations exposed to specific stressors, including combat veterans ${ }^{7}$ and civilians, ${ }^{8}$ have also reported higher
Correspondence: Consuelo Arbona Department of Psychological, Health, and Learning Sciences, University of Houston, 3657 Cullen Boulevard, Room 49I, Houston, TX 77204-5023, USA

Tel +I 7I374398I4

Email carbona@uh.edu 
prevalence rates and more severe symptoms of PTSD among African Americans compared with their non-Hispanic White counterparts. ${ }^{9}$

Researchers have proposed that African Americans' higher levels of exposure to life stressors, including impoverished and violent neighborhoods and racial discrimination, ${ }^{5,10}$ may explain their relatively higher levels of prevalence and severity of PTSD symptoms compared to non-Hispanic Whites. However, differences in the psychometric properties of PTSD assessments, including the latent structure of PTSD symptom clusters, may also explain the observed ethnic group differences in PTSD symptoms. Therefore, to meaningfully compare the prevalence of PTSD rates among African Americans with that among members of other ethnic groups ${ }^{11}$ and accurately interpret cultural correlates of observed group differences, it is necessary to first establish the factorial structure and external validity of PTSD factor scores among African Americans. ${ }^{12,13}$

\section{Factor structure of PTSD symptom clusters}

The Diagnostic and Statistical Manual of Mental Disorders, 4th ed. (DSM-IV) defined PTSD in terms of 17 symptoms organized in three clusters labeled as follows: $\mathrm{B}$, reexperiencing; $\mathrm{C}$, effortful avoidance; and $\mathrm{D}$, emotional numbing. ${ }^{3}$ However, many studies have shown that the original three-factor structure does not provide a good fit for PTSD symptom assessments. ${ }^{14}$ Instead, researchers concluded that two four-factor models labeled emotional numbing (proposed by King et $\mathrm{al}^{15}$ ) and dysphoria (proposed by Simms et $\mathrm{al}^{16}$ ) provide a better fit for the configuration of PTSD symptoms compared to the DSM-IV three-factor model.

In the first modification of the DSM-IV PTSD threefactor configurations, King et $\mathrm{al}^{15}$ developed the emotional numbing model by separating the items in the avoidance cluster $(\mathrm{C} 1-\mathrm{C} 7)$ into two factors labeled avoidance $(\mathrm{C} 1-\mathrm{C} 2)$ and numbing (C3-C7). This change was based on empirical findings suggesting that the two sets of symptoms loaded on different factors and were differentially related to treatment response. ${ }^{17}$ In their four-factor dysphoria model, Simms et $\mathrm{al}^{16}$ retained the separate two-item avoidance cluster (C1-C2), but they grouped the items D1-D3 (sleep difficulty, irritability, and concentration problems) with the remaining numbing items (C3-C7) to create an eight-item dysphoria cluster that reflects general distress. However, most recently, Elhai et a ${ }^{18}$ proposed a five-factor model, labeled dysphoric arousal, that includes the same reexperiencing, avoidance, and numbing factors as the King et al's ${ }^{15}$ emotional numbing model, but separates the King et al's ${ }^{16}$ hyperarousal factor into two clusters labeled dysphoric arousal (D1-D3) and anxious arousal (D4-D5). Elhai et $\mathrm{al}^{18}$ reasoned that items D1-D3 capture symptoms of agitated/restless dysphoria, which are conceptually and empirically distinct from both the numbing (C3-C7) and the anxious arousal (D4-D5) symptoms. A mapping of the items for the three-factor models is provided in Table 1.

In a recent extensive literature review, Armour et al ${ }^{19}$ concluded that while the two four-factor PTSD models proposed by King et $\mathrm{al}^{15}$ and Simms et $\mathrm{al}^{16}$ have received strong empirical support among adult samples, none of the two models have demonstrated a clear superior fit across a majority of studies. In contrast, Elhai et al's ${ }^{18}$ five-factor model has consistently provided the best fit for the latent structure of the 17 PTSD DSM-IV symptoms among samples that have differed in terms of age, nationality, sex, and type of trauma. ${ }^{19}$ However, in most of the studies conducted in the US, participants have been predominantly Caucasians. In one study that examined the latent structure of PTSD symptoms in an African American sample, researchers tested only the goodness of fit of the DSM-IV original three-factor configuration of PTSD symptoms, which yielded a poor fit. ${ }^{20}$ Therefore, it seems safe to conclude that no study has been conducted to examine the relative goodness of fit of the identified best-fitting PTSD factor models with exclusively African American adults. To address this gap in the literature, we compared the relative fit of the three well-established

Table I Mapping of items for each factor model

\begin{tabular}{llll}
\hline PTSD symptoms & $\begin{array}{l}\text { Emotional } \\
\text { numbing }\end{array}$ & $\begin{array}{l}\text { Dysphoria } \\
\text { BI: Intrusive thoughts }\end{array}$ R & $\begin{array}{l}\text { Dysphoric } \\
\text { arousal }\end{array}$ \\
B2: Nightmares & $\mathrm{R}$ & $\mathrm{R}$ & $\mathrm{R}$ \\
B3: Flashbacks & $\mathrm{R}$ & $\mathrm{R}$ & $\mathrm{R}$ \\
B4: Emotional reactivity & $\mathrm{R}$ & $\mathrm{R}$ & $\mathrm{R}$ \\
B5: Physical reactivity & $\mathrm{R}$ & $\mathrm{R}$ & $\mathrm{R}$ \\
C1: Avoidance of thoughts & $\mathrm{A}$ & $\mathrm{A}$ & $\mathrm{R}$ \\
C2: Avoidance of reminders & $\mathrm{A}$ & $\mathrm{A}$ & $\mathrm{A}$ \\
C3: Amnesia for aspects & $\mathrm{N}$ & $\mathrm{D}$ & $\mathrm{N}$ \\
C4: Loss of interest & $\mathrm{N}$ & $\mathrm{D}$ & $\mathrm{N}$ \\
C5: Feeling distant & $\mathrm{N}$ & $\mathrm{D}$ & $\mathrm{N}$ \\
C6: Feeling numb & $\mathrm{N}$ & $\mathrm{D}$ & $\mathrm{N}$ \\
C7: Foreshortened future & $\mathrm{N}$ & $\mathrm{D}$ & $\mathrm{N}$ \\
DI: Sleep disturbance & $\mathrm{H}$ & $\mathrm{D}$ & $\mathrm{DA}$ \\
D2: Irritability & $\mathrm{H}$ & $\mathrm{D}$ & $\mathrm{DA}$ \\
D3: Difficulty concentrating & $\mathrm{H}$ & $\mathrm{D}$ & $\mathrm{DA}$ \\
D4: Hypervigilance & $\mathrm{H}$ & $\mathrm{H}$ & $\mathrm{AA}$ \\
D5: Exaggerated startle & $\mathrm{H}$ & $\mathrm{H}$ & $\mathrm{AA}$ \\
\hline N
\end{tabular}

Notes: $A$, avoidance; $D$, dysphoria; $H$, hyperarousal; $N$, numbing; $R$, reexperiencing. Abbreviations: AA, anxious arousal; DA, dysphoric arousal; PTSD, posttraumatic stress disorder. 
PTSD DSM-IV factorial models among African American male firefighters.

\section{Specificity of PTSD factors}

PTSD symptoms have shown relatively high levels of comorbidity with other disorders among individuals who have experienced different types of trauma, ${ }^{21-23}$ including men in the general population. ${ }^{24}$ In practical terms, truly distinct factors should demonstrate differential correlations with external variables. ${ }^{25}$ Therefore, in addition to testing the factorial structure of PTSD symptoms, researchers have examined the identified factors in relation to symptoms of other disorders. Studies have reported mixed findings regarding the extent to which PTSD symptom clusters show differential associations with depression and alcohol abuse.

In a meta-analysis of 41 studies, Gootzeit and Markon ${ }^{21}$ concluded that, compared to the other symptom clusters, the dysphoria factor in the Simms et al's ${ }^{16}$ dysphoria model showed the strongest correlations with depression, substance abuse, anxiety, and panic. These findings are consistent with Simms et al's ${ }^{16}$ proposition that the dysphoria symptom cluster captures general distress and helps explain the high comorbidity of PTSD with other disorders. However, in other studies, the dysphoria factor showed similar associations with measures of negative emotionality relative to the numbing or hyperarousal factors. ${ }^{26-28}$ The numbing and hyperarousal factors in the King et al's ${ }^{15}$ emotional numbing model also demonstrated similar correlations with depression and alcohol abuse. ${ }^{29}$

Two of the few studies that examined the relative contribution of the five symptom clusters included in the dysphoric arousal model ${ }^{18}$ reported that the dysphoric arousal and numbing clusters were equally related to depression ${ }^{29}$ and alcohol abuse, ${ }^{30}$ while one study indicated that depression was more strongly related to the dysphoric arousal than to the numbing factor. ${ }^{31}$ In several studies, both depression ${ }^{29,32}$ and alcohol abuse $\mathrm{e}^{30}$ were more strongly associated with dysphoric arousal than to anxious arousal, which provides support for Elhai et al's ${ }^{15}$ decision to split the original hypervigilance symptom clusters (items D1-D5) into two factors, dysphoric arousal (D1-D3) and anxious arousal (D4-D5).

In sum, findings have been mixed regarding the level of differentiation that symptom clusters in the competing PTSD models demonstrate in relation to symptoms of other disorders. Therefore, we also examined with African American participants the convergent and discriminant validity of the symptom clusters included in the best-fitting factor model(s) in relation to depression and alcohol dependence symptoms.

\section{The current study}

The objectives of this study were to 1) examine to what extent each of the three competing models, emotional numbing, dysphoria, and dysphoric arousal, provide a good fit for PTSD-C17 items among study participants, 2) compare the goodness of fit of the three PTSD competing factor models, and 3 ) examine the convergent and discriminant validity of the best-fitting PTSD factor model(s) in relation to measures of depression and alcohol dependence symptoms. Based on previous findings, we hypothesized that the five-factor dysphoric arousal model proposed by Elhai et al ${ }^{18}$ would demonstrate the best fit, but we made no predictions regarding the relative fit of the two four-factor models. Because of mixed findings in previous studies, no hypothesis was formulated regarding the convergent and discriminant validity of symptom clusters within the best-fitting model(s).

\section{Methods \\ Participants/procedures}

Participants included were 490 firefighters who self-identified as African Americans and were employed by a large fire department in a major metropolitan city in the southwest US. As part of a department-wide suicide prevention program, participants completed a voluntary paper-and-pencil mental health needs assessment survey that included questions regarding PTSD symptoms, depression, and substance use. Data from African American female firefighters $(n=13)$ were excluded from the analyses, because they represented a very small proportion of the sample (2.6\%), resulting in an effective sample of 477 African American male firefighters.

The study was reviewed and approved by the University of Houston's Institutional Review Board. Participation in the survey was voluntary and the data was gathered anonymously with participants' consent.

\section{Instrumentation}

The PTSD Checklist - Civilian Version (PCL-C17) ${ }^{33}$ was used to assess the 17 posttraumatic stress symptoms identified in the DSM-IV. The PCL-C17, a self-administered screening instrument, asks respondents to consider the "list of problems and complaints that people sometimes have in response to stressful experiences" and to indicate how much they "have been bothered by each problem in the past month" in a Likert-type scale ranging from 1 (not at all) to 5 (extremely). Exposure to specific types of trauma was not assessed in the survey. Possible scores on the PCL-C17 range from 17 to 85 . Based on an extensive review of the literature, Wilkins et $\mathrm{al}^{34}$ concluded that the PCL-C17 has demonstrated sound psychometric properties including test-retest reliability (coefficients ranging from 0.75 
to 0.88 ), internal consistency (coefficients for the total score ranging from 0.83 to 0.94 ), and convergence validity with scores in other PTSD measures (coefficients ranging from 0.63 to 0.90$)$. In the current study, Cronbach's internal reliability coefficient for the PCL-C17 scale's total score was $\alpha=0.94$.

The Patient Health Questionnaire (PHQ-9), ${ }^{35}$ an instrument designed to assess nine symptoms based on the DSM-IV diagnostic criteria for major depressive disorder, was used to assess symptoms of depression. Participants were asked to indicate on a scale from 0 (not at all) to 3 (several days) the number of days during the previous 2 weeks that they were bothered by each symptom (eg, feeling down, depressed, or hopeless). Scores may range from 0 to 27 . PHQ-9 scores with primary care populations have shown good internal reliability (Cronbach's $\alpha$ of 0.89 and 0.86 ) and test-retest reliability over 48 hours $(\kappa=0.48)$, and have discriminated well between people with and without major depression. ${ }^{35}$ The internal reliability coefficient for scores in the PHQ-9 with study participants was 0.85 .

Alcohol dependence was assessed using the Rapid Alcohol Problems Screen (RAPS-4), ${ }^{36}$ a four-item screening instrument, that asks about the presence of four problem behaviors associated with alcohol abuse in the past year: remorse, amnesia, performance, and starter drinking behavior. With a hospital's emergency room sample, a positive response to any of the four items in the RAPS-4 demonstrated a sensitivity of $93 \%$ and specificity of $87 \%$ for alcohol dependence across sex and ethnic subgroups. ${ }^{36}$ In the current study, participants responded yes or no to each question, and the yes responses were summed. Total scores could range from 0 to 4, with higher scores indicating higher levels of substance abuse or dependence. The internal reliability coefficient for scores in the RAPS-4 with study participants was 0.65 .

\section{Plan of analyses}

Confirmatory factor analyses (CFA) were performed in Mplus 7 to test the goodness of fit of each of the three PCL-C17 competing models (objective 1): emotional numbing, ${ }^{15}$ dysphoria, ${ }^{16}$ and dysphoric arousal. ${ }^{18}$ The robust maximum likelihood method, which corrects for non-normality and yields the scaled SatorraBentler (SB) $\chi^{2}$ statistic, was used to assess the model-data fit. ${ }^{37-39}$ To address the study's second objective, the Bayesian information criterion (BIC) $)^{40}$ was used to compare the goodness of fit of the non-nested models, emotional numbing and dysphoria. The SB scaled difference $\chi^{2}$ test ${ }^{41}$ was used to compare the nested models: the dysphoric arousal model was compared to both the emotional numbing and dysphoria models.

To address the study's third objective regarding the discriminant validity of the competing PTSD models in relation to other psychological disorders, three additional CFA were conducted to examine the goodness of fit of expanded competing PTSD model(s) that also included depression and substance dependence latent variables. The relative strength of the observed correlations of PTSD factors with depression and alcohol dependence symptoms was assessed to examine the degree of overlap/specificity of PTSD symptom clusters with the other two disorders.

The following five fit indices were used to determine the model-data fit: the comparative fit index, Tucker-Lewis index (TLI), standardized root mean square residual, and root mean square error of approximation along with its associated $90 \%$ confidence interval. As recommended by $\mathrm{Hu}$ and Bentler, ${ }^{42}$ cutoffs for acceptable model fit were comparative fit index $\geq 0.90$ ( $\geq 0.95$, excellent fit), TLI $\geq 0.90$ ( $\geq 0.95$, excellent fit), standardized root mean square residual $\leq 0.10(\leq 0.08$, excellent fit), and root mean square error of approximation $\leq 0.08(\leq 0.06$, excellent fit), and at least two of these fit indices were examined in combination to assess the model-data fit.

\section{Results \\ Descriptive statistics}

As shown in Table 2, participants were evenly distributed in terms of age and years of service in the fire department; the majority were married or lived with a partner. In terms of education, most of the participants reported completion of some postsecondary schooling and close to half of them reported either a 2- or 4-year college degree. The observed mean score on the PCL-C17 for the total sample was 24.81 ( $\mathrm{SD}=10.18$ ). Using a cutoff score of 39 , as suggested by

Table 2 Participants' demographics

\begin{tabular}{lll}
\hline Characteristics & $\mathbf{N}$ & $\%$ \\
\hline Age (years) & 138 & 29 \\
19-34 & 162 & 34 \\
$35-44$ & 172 & 36 \\
$45-66$ & & \\
Education level & 29 & 6.1 \\
$\quad$ High school & 230 & 48.2 \\
$\quad$ Some college & 215 & 45.1 \\
$\quad$ College graduate & & \\
Marital status & 330 & 71.3 \\
$\quad$ Married/with partner & 147 & 28.7 \\
$\quad$ Not married & 135 & 28.3 \\
Military service (yes) & & \\
Years of service in fire department & 131 & 27.5 \\
5 or less & 187 & 39.2 \\
6-20 & 159 & 33.3 \\
21 or more &
\end{tabular}

Note: Numbers within categories may not add up to presented $\mathrm{N}$ due to missing values. 
Table 3 Fit statistics for CFA of PCL factor models

\begin{tabular}{|c|c|c|c|c|c|c|c|c|c|}
\hline Models & SB $\chi^{2}$ & $d f$ & Scaling factor & CFI & TLI & SRMR & RMSEA & RMSEA $90 \% \mathrm{Cl}$ & BIC \\
\hline \multicolumn{10}{|l|}{ PTSD four- and five-factor models } \\
\hline Emotional numbing & 198.195 & 113 & I.87I & 0.967 & 0.960 & 0.033 & 0.040 & $0.030-0.049$ & $15,370.55$ \\
\hline Dysphoria & 232.277 & 113 & 1.878 & 0.954 & 0.944 & 0.037 & 0.047 & $0.038-0.056$ & $15,435.85$ \\
\hline Dysphoric arousal & 195.160 & 109 & 1.883 & 0.966 & 0.958 & 0.032 & 0.041 & $0.031-0.050$ & $|5,39| .74$ \\
\hline \multicolumn{10}{|c|}{ Expanded PTSD four- and five-factor models incorporating external correlates: depression and alcohol dependence } \\
\hline Expanded emotional numbing & 754.468 & 390 & 1.636 & 0.914 & 0.905 & 0.052 & 0.044 & $0.040-0.049$ & $21,111.34$ \\
\hline Expanded dysphoria & 794.321 & 390 & 1.639 & 0.905 & 0.894 & 0.053 & 0.047 & $0.042-0.051$ & $21,178.32$ \\
\hline Expanded dysphoric arousal & 748.459 & 384 & 1.639 & 0.914 & 0.903 & 0.051 & 0.045 & $0.040-0.049$ & $21,140.01$ \\
\hline
\end{tabular}

Abbreviations: BIC, Bayesian information criterion; CFA, confirmatory factor analyses; CFI, comparative fit index; $\mathrm{Cl}$, confidence interval; df, degrees of freedom; RMSEA, root mean square error of approximation; PCL, PTSD checklist; PTSD, posttraumatic stress disorder; SB $\chi^{2}$, Satorra-Bentler chi-square statistic; SRMR, standardized root mean square residual; TLI, Tucker-Lewis index.

Chiu et $\mathrm{al}^{43}$ in a study with firefighters, $40(8.4 \%)$ participants demonstrated elevated PTSD risk.

\section{Factor structure of PTSD symptoms}

Model fit statistics for the CFA performed to test the goodness of fit of each of the three PCL-C17 competing models are provided in Table 3. According to the earlier mentioned criteria, the three PTSD factor models showed good to excellent fit. The fit indices for the emotional numbing and the dysphoric arousal models were in the excellent range. For the dysphoria model, only the TLI value (0.944) was slightly below the excellent fit threshold (0.95). However, the $\Delta \mathrm{BIC}$ of 65.30 indicates that the emotional numbing model fit better than the dysphoria model (among non-nested models, a BIC difference $>10$ indicates very strong support for the model with the lower BIC value ${ }^{44}$ ). Next, the fit of the five-factor dysphoric arousal model was compared to each of the two four-factor models. SB scaled difference $\chi^{2}$ tests revealed that the five-factor dysphoric arousal model fit significantly better than the four-factor dysphoria model $\left(\chi^{2}=39.3095, d f=4\right.$, $P<0.001)$ and that there was no significant difference in the goodness of fit between the five-factor dysphoric arousal and the four-factor emotional numbing models $\left(\chi^{2}=2.2308\right.$, $d f=4, P>0.05$ ). In sum, results indicated that both the emotional numbing and the dysphoric arousal models similarly provided an excellent representation of the latent structure of the PTSD factors. In the three PTSD models, all items loaded significantly in their corresponding factors (Table 4).

\section{Relationships with external measures of depression and alcohol dependence}

To examine the convergent and discriminant validity of the factors, we built three additional factor models, one for each of the PTSD models, by adding the latent depression and alcohol dependence factors represented by the items in the PCQ-9 and the RASP-4, respectively. The model-data fit of each of the three expanded PTSD models was examined using
Table 4 Standardized factor loadings of PTSD symptoms for competing PTSD models

\begin{tabular}{|c|c|c|c|}
\hline \multirow[t]{2}{*}{ Items } & \multicolumn{3}{|l|}{ PTSD models } \\
\hline & $\begin{array}{l}\text { Emotional } \\
\text { numbing }\end{array}$ & Dysphoria & $\begin{array}{l}\text { Dysphoric } \\
\text { arousal }\end{array}$ \\
\hline & Re-experiencing & Re-experiencing & Re-experiencing \\
\hline $\begin{array}{l}\text { BI: Intrusive } \\
\text { thoughts }\end{array}$ & 0.742 & 0.738 & 0.741 \\
\hline B2: Nightmares & 0.784 & 0.779 & 0.783 \\
\hline B3: Flashbacks & 0.842 & 0.844 & 0.843 \\
\hline $\begin{array}{l}\text { B4: Emotional } \\
\text { reactivity }\end{array}$ & 0.832 & 0.837 & 0.832 \\
\hline \multirow[t]{2}{*}{$\begin{array}{l}\text { B5: Physical } \\
\text { reactivity }\end{array}$} & 0.784 & 0.782 & 0.784 \\
\hline & Avoidance & Avoidance & Avoidance \\
\hline $\begin{array}{l}\mathrm{Cl} \text { : Avoidance } \\
\text { thoughts }\end{array}$ & 0.835 & 0.833 & 0.835 \\
\hline \multirow[t]{2}{*}{$\begin{array}{l}\text { C2: Avoidance } \\
\text { reminders }\end{array}$} & 0.844 & 0.845 & 0.844 \\
\hline & Numbing & Dysphoria & Numbing \\
\hline $\begin{array}{l}\text { C3: Amnesia for } \\
\text { aspects }\end{array}$ & 0.698 & 0.677 & 0.696 \\
\hline C4: Loss of interest & 0.772 & 0.752 & 0.773 \\
\hline C5: Feeling distant & 0.766 & 0.756 & 0.767 \\
\hline C6: Feeling numb & 0.834 & 0.820 & 0.835 \\
\hline C7: Foreshortened & 0.811 & 0.809 & 0.810 \\
\hline \multicolumn{4}{|l|}{ future } \\
\hline & Hyperarousal & & $\begin{array}{l}\text { Dysphoric } \\
\text { arousal }\end{array}$ \\
\hline $\begin{array}{l}\text { DI: Sleep } \\
\text { disturbance }\end{array}$ & 0.592 & 0.564 & 0.585 \\
\hline D2: Irritability & 0.762 & 0.711 & 0.751 \\
\hline \multirow[t]{2}{*}{$\begin{array}{l}\text { D3: Difficulty } \\
\text { concentration }\end{array}$} & 0.730 & 0.722 & 0.724 \\
\hline & & Hyperarousal & Anxious arousal \\
\hline D4: Hypervigilance & 0.581 & 0.571 & 0.576 \\
\hline $\begin{array}{l}\text { D5: Exaggerated } \\
\text { startle }\end{array}$ & 0.788 & 0.788 & 0.781 \\
\hline
\end{tabular}

Note: All factor loadings were statistically significant at $P<0.001$.

Abbreviation: PTSD, posttraumatic stress disorder.

CFA procedures as described earlier. As shown in Table 3, the correlated six- and seven-factor expanded emotional numbing and dysphoric arousal models demonstrated good to excellent fit. In contrast, the TLI for the expanded dysphoria model 
(TLI $=0.894)$ was slightly below the threshold ( 0.90$)$ for good fit. The $\triangle \mathrm{BIC}$ of 66.98 between the two non-nested models indicated that the expanded emotional numbing model fit better than the expanded dysphoria model. ${ }^{44} \mathrm{SB}$ scaled difference $\chi^{2}$ tests revealed that the expanded dysphoric arousal model fit significantly better than the expanded dysphoria model $\left(\chi^{2}=46.5425, d f=6, P<0.001\right)$ and that there was no significant difference in the goodness of fit between the expanded dysphoric arousal and emotional numbing models $\left(\chi^{2}=5.3098, d f=6, P>0.05\right)$. These findings suggest that the PTSD symptom clusters included in both the four-factor emotional numbing and the five-factor dysphoric arousal models are distinguishable from depression and anxiety symptoms at the factor level.

Next, we examined to what extent the dysphoric arousal, anxious arousal, and numbing symptoms in the dysphoric arousal model ${ }^{18}$ and the dysphoria symptoms in the dysphoria model $^{16}$ showed differential associations with depression and alcohol dependence. These comparisons were selected because the main difference between the three competing factor models is the placement of items D1-D3. In the emotional numbing model, ${ }^{15}$ items D1-D5 comprise the hyperarousal factor, while in the dysphoric arousal model ${ }^{18}$ items D1-D5 are split into two factors: dysphoric arousal (D1-D3) and anxious arousal (D4-D5). In the dysphoria model, ${ }^{16}$ items D1-D3 are combined with items C3-C7 to form the dysphoria cluster and items D4-D5 form that model's hyperarousal cluster. Steiger's $z$-tests were used to identify statistically significant differences between the compared correlation coefficients. $^{45}$

As shown in Table 5, correlations of PTSD factors with depression symptoms were relatively high (ranging from $r=0.44$ to 0.65 ). Results of Steiger's $z$-tests ${ }^{45}$ showed that among the dysphoric arousal symptom clusters, the difference in the relation of depression to numbing $(r=0.59)$ versus

Table 5 Correlation of PTSD symptom clusters, depression, and alcohol dependence

\begin{tabular}{llllllll}
\hline Variables & $\mathbf{I}$ & $\mathbf{2}$ & $\mathbf{3}$ & $\mathbf{4}$ & $\mathbf{5}$ & $\mathbf{6}$ & $\mathbf{7}$ \\
\hline Reexperiencing $^{\mathrm{a}, \mathrm{b}, \mathrm{c}}$ & & & & & & & \\
Avoidance $^{\mathrm{a}, \mathrm{b}, \mathrm{c}}$ & 0.70 & & & & & & \\
Numbing, $^{\mathrm{a}, \mathrm{c}}$ & 0.68 & 0.69 & & & & & \\
Dysphoric arousa $^{\mathrm{a}, \mathrm{c}}$ & 0.64 & 0.57 & 0.72 & & & & \\
Anxious arousal $^{\mathrm{b}, \mathrm{c}}$ & 0.58 & 0.53 & 0.63 & 0.67 & & & \\
Dysphoria $^{\mathrm{b}}$ & 0.71 & 0.69 & 0.95 & 0.89 & 0.69 & & \\
Depression $_{\text {Alcohol dependence }}$ & 0.55 & 0.44 & 0.59 & 0.63 & 0.52 & 0.65 & \\
\hline Notes & 0.26 & 0.25 & 0.26 & 0.20 & 0.29 & 0.27 \\
\hline
\end{tabular}

Notes: ${ }^{a}$ Emotional numbing model; bdysphoria model; ${ }^{\mathrm{c}}$ dysphoric arousal model. All correlations were statistically significant at $P<0.00 \mathrm{I}$.

Abbreviation: PTSD, posttraumatic stress disorder. the relation of depression to dysphoric arousal $(r=0.63)$ was not statistically significant, $z=-1.54, P=0.12$. However, depression was more strongly correlated with both numbing $(r=0.59, z=2.23, P=0.03)$ and dysphoric arousal symptoms $(r=0.63 ; z=3.79, P<0.001)$ than to anxious arousal $(r=0.52)$ symptoms. Results also showed that depression was more strongly correlated with the dysphoria symptom cluster included in the Simms' dysphoria model $(r=0.65)$ than to the hyperarousal/anxious arousal cluster included in both the Simms' dysphoria and the Elhai's dysphoric arousal models ( $r=0.52 ; z=4.67, P<0.001)$. This last finding makes sense, since the Simms et al's ${ }^{16}$ dysphoria cluster combines the items from the five-factor numbing (C3-C7) and dysphoric arousal (D1-D3) clusters, both of which were more strongly related to depression compared to the anxious arousal cluster (D4-D5).

In contrast to findings regarding depression, the correlations of PTSD symptom clusters included in the dysphoric arousal model with alcohol dependence were relatively low (ranging from $r=0.20$ to 0.26 ). Differences in the correlations of alcohol dependence with emotional numbing $(r=0.25)$ versus dysphoric arousal $(r=0.26 ; z=0.302, P>0.05)$ or anxious arousal ( $r=0.20 ; z=1.31, P>0.05)$ were not statistically significant. Similarly, alcohol dependence was not differentially related to dysphoric arousal $(r=0.26)$ versus anxious arousal $(r=0.20 ; z=1.66, P>0.05)$. Similar to results with depression, alcohol dependence was more strongly correlated with the dysphoria symptom cluster included in the dysphoria model $(r=0.27)$ than to the hyperarousal/anxious arousal clusters included in the dysphoria and dysphoric arousal models $(r=0.20 ; z=2.01, P=0.04)$.

\section{Discussion}

The purpose of this study was to examine among African American male firefighters the relative fit of three competing factor models of PTSD symptoms that have been established with other populations: the two four-factor emotional numbing ${ }^{15}$ and dysphoria models ${ }^{16}$ and the more recently proposed five-factor dysphoric arousal model. ${ }^{18}$ Consistent with a previous study by Armour et al, ${ }^{19}$ the three PTSD factor models demonstrated good to excellent fit. In terms of relative fit of the three models, comparison between the two four-factor models using the BIC criteria ${ }^{44}$ indicated that the emotional numbing model demonstrated a superior fit to the data than the dysphoric arousal model. Results of $\chi^{2}$ difference tests showed that while the five-factor dysphoric arousal model demonstrated a superior fit compared to the four-factor dysphoria model, it did not show a superior fit compared to the four-factor emotional numbing model, as 
was hypothesized. These findings differ from previous studies that have consistently shown that the five-factor model demonstrates a superior fit than the two well-established fourfactor models. ${ }^{29,31,32,46}$ Therefore, results provided support for the study's first hypothesis and partial support for the second hypothesis. In sum, both the four-factor emotional numbing and the five-factor dysphoric arousal models emerged as the best-fitting models for the structure of PTSD symptoms among African American male firefighters.

Researchers have noted that in addition to identifying the best-fitting structural models, it is also important to examine the construct validity of the identified PTSD clusters in relation to symptoms of other disorders. ${ }^{31,47} \mathrm{~A}$ second set of CFA that added a depression and a substance dependence factor to the respective PTSD competing models yielded acceptable fit for the dysphoria model and good fit for both the emotional numbing and the dysphoric arousal models. These findings indicate that the respective PTSD clusters are distinguishable from depression and anxiety symptoms at the factor level, which provides support for the construct validity of the two best-fitting models with African American participants. Studies with populations in the US, Asia, and Europe have reported similar findings. ${ }^{29,31,32}$

Results of the two sets of CFA provided statistical support for the two factor models that represent PTSD in terms of reexperiencing, avoidance, numbing, and hyperarousal factors. The only difference between the two best-fitting models is the separation of the five items (D1-D5) included in the King et al's ${ }^{15}$ emotional numbing model's hyperarousal cluster into two arousal factors in the Elahi et al's ${ }^{18}$ dysphoric arousal model, dysphoric arousal (items D1-D3) and anxious arousal (D3-D5). Examination of the relative specificity of PTSD clusters included in the dysphoric arousal model in relation to depression showed that the difference in the correlations of the emotional numbing (items $\mathrm{C} 3-\mathrm{C} 7$ ) and the dysphoric arousal (items D1-D3) clusters with depression was not statistically significant, which provides support for combining both sets of symptoms into one factor, as proposed in the dysphoria cluster included in the Simms et al's ${ }^{16}$ dysphoria model. At the same time, depression was more strongly associated with both the emotional numbing and dysphoric arousal clusters than with anxious arousal, which provides support for Elhai et al's ${ }^{18}$ decision to separate the hyperarousal symptom cluster (items D1-D5 in the emotional numbing model) into two factors, dysphoric arousal (D1-D3) and anxious arousal (D4-D5). Further analyses also revealed that within the dysphoria model ${ }^{16}$ depression was more strongly related to the dysphoria cluster (items C3-C7, D1-D3) than to the hyperarousal cluster (items D4-D5, same items as in anxious arousal cluster in the five-factor dysphoric arousal model).

In contrast to the findings regarding depression, the correlations of alcohol dependence with each of the five PTSD clusters included in the dysphoric arousal model were relatively low (ranging from $r=0.20$ to 0.26 ), and the five clusters were equally correlated with alcohol dependence. The only two clusters that showed a differential relation to alcohol dependence were the dysphoria cluster included in the dysphoria four-factor model versus the two-item (D4-D5) hyperarousal/anxious arousal cluster included in both the dysphoria and the dysphoric arousal models. Alcohol dependence was more strongly related to the dysphoria symptom cluster $(r=0.27)$ than to the hyperarousal/anxious arousal cluster $(r=0.20)$. Even though correlations were relatively weak, this finding provides support for the specificity of the anxious arousal symptom cluster to PTSD in relation to alcohol dependence.

As noted by Armour et al, ${ }^{31}$ findings regarding the relation of PTSD clusters to depression and alcohol dependence suggest that, even though the emotional numbing ${ }^{15}$ and the dysphoric arousal ${ }^{18}$ models provided a superior fit based on BIC and $\chi^{2}$ differences' testing, the dysphoria four-factor model $^{18}$ may be a more parsimonious representation of PTSD's latent structure than the five-cluster model. The dysphoria symptom cluster (dysphoria model), which combines the items from two dysphoric arousal clusters (numbing and dysphoric arousal factors), seems to best capture PTSD symptoms that reflect general distress. ${ }^{46}$ At the same time, the dysphoria model includes the anxious arousal cluster (items D4-D5), which has consistently shown to be less strongly related to depression and alcohol dependence (and, therefore, more specific to PTSD) than the dysphoric arousal cluster (items D1-D3). ${ }^{29,30,32}$ Researchers have noted that hyperarousal symptoms D1-D5 may play an important role in the progression of PTSD and related functional difficulties. ${ }^{48}$ Therefore, identifying hyperarousal symptoms that are differentially related to other disorders may allow researchers and clinicians to devise specific intervention strategies tailored to each type of symptom and enhance treatment effectiveness. ${ }^{32}$

Findings must be interpreted taking into account the study's limitations. Data regarding PTSD symptoms were collected via a self-report measure. It is possible that data from clinicianrated PTSD measures may yield different results regarding factor invariance across groups. ${ }^{49}$ Even though it is reasonable to assume that firefighters are exposed to traumatic events 
due to the nature of their daily work, information regarding participants' specific traumatic experiences was not obtained as part of the study. Findings from Elhai et $\mathrm{al}^{50}$ suggest that both trauma experience and the extent to which participants rate PTSD symptoms with reference to a most traumatic event or not may moderate findings regarding the structure of PTSD symptoms. In addition, it is not known how the study results will generalize to African American women or women firefighters, since the sample only included male firefighters. Finally, the factor structure of the 20 symptoms included in the DSM-V needs to be examined with African American populations.

\section{Conclusion}

This study extends support for the well-established PTSD symptom factor models to African Americans, a population with whom the structure of the well-established PTSD models had not been examined earlier. Results suggest that scores in the various symptom clusters obtained from the PCL-C17 Civilian PTSD assessment can be meaningfully interpreted in comparisons of the prevalence of PTSD rates between African Americans and members of other ethnic groups and in the examination of cultural correlates of observed group differences.

\section{Disclosure}

The authors report no conflicts of interest in this work.

\section{References}

1. Hogan N, Costello S, Boyle M, Williams B. Measuring workplace trauma response in Australian paramedics: an investigation into the psychometric properties of the Impact of Event Scale. Psychol Res Behav Manag. 2015;8:287-294.

2. Malcoun E, Williams MT, Bahojb-Nouri LV. Assessment of posttraumatic stress disorder in African Americans. In: Benuto LT, Leany BD, editors. Guide to Psychological Assessment with African Americans. New York, NY: Springer; 2015:163-182.

3. American Psychiatric Association. Diagnostic and Statistical Manual of Mental Disorders. 4th ed. Washington, DC: American Psychiatric Press; 2000.

4. Breslau J, Aguilar-Gaxiola S, Kendler KS, Su M, Williams D, Kessler RC. Specifying race-ethnic differences in risk for psychiatric disorder in a USA national sample. Psychol Med. 2006;36(1):57-68.

5. Himle JA, Baser RE, Taylor RJ, Campbell RD, Jackson JS. Anxiety disorders among African Americans, blacks of Caribbean descent, and non-Hispanic whites in the United States. J Anxiety Disord. 2009;23(5):578-590.

6. Roberts AL, Gilman SE, Breslau J, Breslau N, Koenen KC. Race/ ethnic differences in exposure to traumatic events, development of post-traumatic stress disorder, and treatment-seeking for post-traumatic stress disorder in the United States. Psychol Med. 2011;41(1):71-83.

7. Dohrenwend BP, Turner JB, Turse NA, Lewis-Fernandez R, Yager TJ. War-related posttraumatic stress disorder in Black, Hispanic, and majority of White Vietnam veterans: the roles of exposure and vulnerability. J Trauma Stress. 2008;21(2):133-141.

8. Norris FH. Epidemiology of trauma: frequency and impact of different potentially traumatic events on different demographic groups. J Consult Clin Psychol. 1992;60(3):409-418.
9. Hinton DE, Lewis-Fernández R. The cross-cultural validity of posttraumatic stress disorder: implications for DSM-5. Depress Anxiety. 2011;28(9):783-801.

10. Alim TN, Charney DS, Mellman TA. An overview of posttraumatic stress disorder in African Americans. J Clin Psychol. 2006;62(7):801-813.

11. Meredith W, Teresi JA. An essay on measurement and factorial invariance. Med Care. 2006;44(11 suppl 3):S69-S77.

12. Cheung GW, Lau RS. A direct comparison approach for testing measurement invariance. Organ Res Methods. 2012;15(2):167-198.

13. Contractor AA, Claycomb MA, Byllesby BM, et al. Hispanic ethnicity and Caucasian race: relations with posttraumatic stress disorder's factor structure in clinic-referred youth. Psychol Trauma. 2015;7(5): 456-464.

14. Yufik T, Simms LJ. A meta-analytic investigation of the structure of posttraumatic stress disorder symptoms. J Abnorm Psychol. 2010;119(4):764-776.

15. King DW, Leskin GA, King LA, Weathers FW. Confirmatory factor analysis of the Clinician-Administered PTSD Scale: evidence for the dimensionality of posttraumatic stress disorder. Psychol Assess. 1998;10(2):90-96.

16. Simms LJ, Watson D, Doebbeling BN. Confirmatory factor analyses of posttraumatic stress symptoms in deployed and nondeployed veterans of the Gulf War. J Abnorm Psychol. 2002;111(4):637-647.

17. Asmundson GJ, Stapleton JA, Taylor S. Are avoidance and numbing distinct PTSD symptom clusters? J Trauma Stress. 2004;17(6): 467-475.

18. Elhai JD, Biehn TL, Armour C, Klopper JJ, Frueh BC, Palmieri PA. Evidence for a unique PTSD construct represented by PTSD's D1-D3 symptoms. J Anxiety Disord. 2011;25(3):340-345.

19. Armour C, Müllerová J, Elhai JD. A systematic literature review of PTSD's latent structure in the diagnostic and statistical manual of mental disorders: DSM-IV to DSM-5. Clin Psychol Rev. 2016;44:60-74.

20. Kibler JL, Ma MM, Lyons JA, Dollar KM, Brisco K, Banks PG. Psychometric properties of the posttraumatic stress checklist among young African-American men and women. Psychol Trauma Theory Res Pract Policy. 2011;23(1):77-83.

21. Gootzeit J, Markon K. Factors of PTSD: differential specificity and external correlates. Clin Psychol Rev. 2011;31(6):993-1003.

22. Grant DM, Beck JG, Marques L, Paylo SA, Clapp JD. The structure of distress following trauma: posttraumatic stress disorder, major depressive disorder, and generalized anxiety disorder. J Abnorm Psychol. 2008;117(3):662-672.

23. Maguen S, Stalnaker M, McCaslin S, Litz BT. PTSD subclusters and functional impairment in Kosovo peacekeepers. Mil Med. 2009;174(8):779-785.

24. Brady KT, Killeen TK, Brewerton T, Lucerini S. Comorbidity of psychiatric disorders and post-traumatic stress disorder. JClin Psychiatry. 2000;61(suppl 7):22-32.

25. Palmieri PA, Weathers FW, Difede J, King DW. Confirmatory factor analysis of the PTSD checklist and the Clinician-Administered PTSD Scale in disaster workers exposed to the World Trade Center ground zero. J Abnorm Psychol. 2007;116:329-341.

26. Biehn TL, Contractor AA, Elhai JD, et al. Latent dimensions of posttraumatic stress disorder and their relations with alcohol use disorder. Soc Psychiatry Psychiatr Epidemiol. 2016;51(3):421-429.

27. Marshall GN, Schell TL, Miles JNV. All PTSD symptoms are highly associated with general distress: ramifications for the dysphoria symptom cluster. J Abnorm Psychol. 2010;119(1):126-135.

28. Miller MW, Wolf EJ, Harrington KM, Brown TA, Kaloupek DG, Keane TM. An evaluation of competing models for the structure of PTSD symptoms using external measures of comorbidity. J Trauma Stress. 2010;23(5):631-638.

29. Armour C, O'Connor M, Elklit A, Elhai JD. Assessing posttraumatic stress disorder's latent structure in elderly bereaved European trauma survivors. J Nerv Ment Dis. 2013;201(10):901-906.

30. Trautmann S, Schönfeld S, Behrendt S, et al. Associations between lifetime PTSD symptoms and current substance use disorders using a five-factor model of PTSD. J Anxiety Disord. 2015;29:93-100. 
31. Armour C, Elhai JD, Richardson D, Ractliffe K, Wang L, Elklit A. Assessing a five factor model of PTSD: is dysphoria arousal a unique PTSD construct showing differential relationships with anxiety and depression? J Anxiety Disord. 2012;26(2):368-376.

32. Wang L, Li Z, Shi Z, et al. Testing the dimensionality of posttraumatic stress responses in young Chinese adult earthquake survivors: further evidence for "dysphoric arousal" as a unique PTSD construct. Depress Anxiety. 2011;28(12):1097-1104.

33. Weathers FW, Litz BT, Herman DS, Huska JA, Keane TM. The PTSD Checklist (PCL): reliability, validity, and diagnostic utility. Paper presented at: Annual Meeting of the International Society for Traumatic Stress Studies; October 24, 1993; San Antonio, TX.

34. Wilkins KC, Lang AJ, Norman SB. Synthesis of the psychometric properties of the PTSD Checklist (PCL) military, civilian, and specific versions. Depress Anxiety. 2011;28(7):596-606.

35. Blackwell TL, McDermott A. Test review: Patient Health Questionnaire-9 (PHQ-9). Rehabil Couns Bull. 2014;57(4):246-248.

36. Cherpitel CJ. A brief screening instrument for problem drinking in the emergency room: the RAPS4. J Stud Alcohol. 2000;61(3):447-449.

37. Satorra A, Bentler PM. A scaled difference chi-square test statistic for moment structure analysis. Psychometrika. 2001;66(4):507-514.

38. Curran PJ, West SG, Finch JF. The robustness of test statistics to nonnormality and specification error in confirmatory factor analysis. Psychol Methods. 1996;1(1):16-29.

39. Fan W, Hancock GR. Robust means modeling: an alternative for hypothesis testing of independent means under variance heterogeneity and nonnormality. J Educ Behav Stat. 2012;37(1):137-156.

40. Schwarz G. Estimating the dimension of a model. Ann Stat. 1978;6: $461-468$
41. Bryant FB, Satorra A. Principles and practice of scaled difference chisquare testing. Struct Equ Modeling Multidiscipl J. 2012;19(3):372-398.

42. Hu LT, Bentler PM. Cutoff criteria for fit indexes in covariance structure analysis: conventional criteria versus new alternatives. Struct Equ Modeling. 1999;6(1):1-55.

43. Chiu S, Webber MP, Zeig-Owens R, et al. Performance characteristics of the PTSD Checklist in retired firefighters exposed to the World Trade Center disaster. Ann Clin Psychiatry. 2011;23(2):95-104.

44. Raftery AE. Bayesian model selection in social research. Sociol Method. 1995;25:111-163.

45. Steiger JH. Tests for comparing elements of a correlation matrix. Psychol Bull. 1980;87:245-251.

46. Demirchyan A, Goenjian AK, Khachadourian V. Factor structure and psychometric properties of the Posttraumatic Stress Disorder (PTSD) Checklist and DSM-5 PTSD symptom set in a long-term postearthquake cohort in Armenia. Assessment. 2015;22(5):594-606.

47. Elhai JD, Palmieri PA. The factor structure of posttraumatic stress disorder: a literature update, critique of methodology, and agenda for future research. J Anxiety Disord. 2011;25(6):849-854.

48. Heir T, Piatigorsky A, Weisæth L. Posttraumatic stress symptom clusters associations with psychopathology and functional impairment. JAnxiety Disord. 2010;24(8):936-940.

49. Palmieri PA, Marshall GN, Schell TL. Confirmatory factor analysis of posttraumatic stress symptoms in Cambodian refugees. J Trauma Stress. 2007;20(2):207-216.

50. Elhai JD, Engdahl RM, Palmieri PA, Naifeh JA, Schweinle A, Jacobs GA. Assessing posttraumatic stress disorder with or without reference to a single, worst traumatic event: examining differences in factor structure. Psychol Assess. 2009;21:629-634.
Psychology Research and Behavior Management

\section{Publish your work in this journal}

Psychology Research and Behavior Management is an international, peerreviewed, open access journal focusing on the science of psychology and its application in behavior management to develop improved outcomes in the clinical, educational, sports and business arenas. Specific topics covered in the journal include: Neuroscience, memory and decision making; Behavior

\section{Dovepress}

modification and management; Clinical applications; Business and sports performance management; Social and developmental studies; Animal studies. The manuscript management system is completely online and includes a very quick and fair peer-review system, which is all easy to use. Visit http://www. dovepress.com/testimonials.php to read real quotes from published authors. 